\title{
Molecular Modeling Studies on Biochanin-A as a Potential Dual Inhibitor for VEGFR-2 and Cyclin D1-CDK-4 Complex
}

\author{
Mohamed Mahmoud ${ }^{\mathrm{a}}$, Marwa A Ali ${ }^{\mathrm{a}}$, Amgad Albohy ${ }^{\mathrm{b}}$, Suher K Zada ${ }^{\mathrm{c}}$, Mai F Tolba ${ }^{\mathrm{a}, \mathrm{d}}$, Dalal Abou El Ella ${ }^{\mathrm{e}^{*}}$ \\ ${ }^{a}$ Department of Pharmacology and Toxicology, Faculty of Pharmacy, Ain Shams University, Cairo 11566, Egypt \\ ${ }^{\mathrm{b}}$ Department of Pharmaceutical Chemistry, Faculty of Pharmacy, British University in Egypt (BUE), El-Sherouk \\ City, Suez Desert Road, Cairo 11837, Egypt \\ ${ }^{\mathrm{c}}$ Department of Biology, School of Sciences and Engineering, American University in Cairo (AUC), Egypt \\ ${ }^{\mathrm{d}}$ Center of Drug Discovery Research and Development, Faculty of Pharmacy, Ain Shams University, Cairo 11566, \\ Egypt \\ ${ }^{\mathrm{e}}$ Department of Pharmaceutical Chemistry, Faculty of Pharmacy, Ain Shams University, Cairo 11566, Egypt
}

\begin{abstract}
Biochanin-A is a known phytoestrogen that is mainly found in red clover. It has several biological activities including anticancer, anti-inflammatory, and antioxidant effects. Preclinical studies showed that Biochanin-A has anticancer properties in different cancer models. This effect was found to happen through a diversity of mechanisms inducing cell cycle arrest, apoptosis, and antiangiogenic effects. Moreover, despite being a promising nature-derived anticancer agent, there is a paucity of information regarding specific target validation studies for Biochanin-A. In this study, we first predicted the physicochemical properties of Biochanin-A using two different online tools (SwissADME and pkCSM), and then we performed an in silico molecular docking studies for Biochanin-A as a potential dual inhibitor for Cyclin-D1-cyclin-dependent kinase (CDK) 4 complex and vascular endothelial growth factor receptor (VEGFR-2) which are key molecular targets for cancer therapy. The results suggest that Biochanin-A interacts with both Cyclin D1-CDK4 complex and VEGFR-2 with a docking affinity that is comparable to their standard inhibitors. These results open the door for further follow-up investigations.
\end{abstract}

Keywords: Biochanin-A; Cytotoxicity; Docking; ADME; Cyclin-D1-CDK4; VEGFR-2.

*Correspondence | Dalal Abou El Ella; Department of Pharmaceutical Chemistry, Faculty of Pharmacy, Ain Shams University, Cairo 11566, Egypt. Email: dalal@pharma.asu.edu.eg

Citation | Mahmoud M, Ali MA, Albohy A, Zada SK, Tolba MF, Abou El Ella DA, 2021. Molecular Modeling Studies on Biochanin-A as a Potential Dual inhibitor for VEGFR-2 and Cyclin D1-CDK-4 Complex. Arch Pharm Sci ASU 5(1): 16-32

DOI: $10.21608 /$ aps.2021.59204.1050

Print ISSN: 2356-8380. Online ISSN: 2356-8399.

Received 03 February 2021. Accepted 1 April 2021.

Copyright: ${ }^{\circledR} 2021$ Abou El Ella et al. This is an open-access article licensed under a Creative Commons Attribution 4.0 International License (CC BY 4.0), which permits unrestricted use, distribution, and reproduction in any medium, provided the original author(s) and source are credited.

Published by: Ain Shams University, Faculty of Pharmacy

\section{INTRODUCTION}

The use of natural products to treat or control health conditions in humans has increased recently. Flavonoids are one of the most common natural products found in several plant families. Isoflavones (Fig. 1) are a subclass of flavonoids and the main family of phytoestrogens that have undergone an intensive investigation for discovering their beneficial activities towards numerous human diseases, including cancers [1]. Biochanin-A (Fig. 2) is a natural isoflavone that is abundant in red clover (Trifolium pretense), peanuts, alfalfa sprouts, cabbage, and soybean [2, 3]. It is classified as a phytoestrogen due to its structural similarity to estrogens along with its 
capability to interact with estrogen receptors [4]. Epidemiological studies have shown that people receiving high isoflavone intake through soy consumption have a lower incidence of many cancers such as bladder, prostate, colon, and gastric cancer [5]. Numerous studies investigating the anticancer activity of Biochanin-A were performed in different cancer cell lines, followed by in vivo models. Many types of tumors could be suppressed by Biochanin-A, such as lung cancer, prostate cancer, gastrointestinal tract, pancreatic, breast, osteosarcoma, malignant melanoma, and tumors of the central nervous system [6]. Biochanin-A is available in the form of a supplement named Promensil $^{\circledR}$ that is currently marketed in North America. This product contains $43.5 \mathrm{mg}$ of isoflavones, with Biochanin-A representing the major constituent with $26.5 \mathrm{mg}$ of the total tablet content and is mainly indicated for the treatment of postmenopausal symptoms as osteoporosis and hot flashes [7].

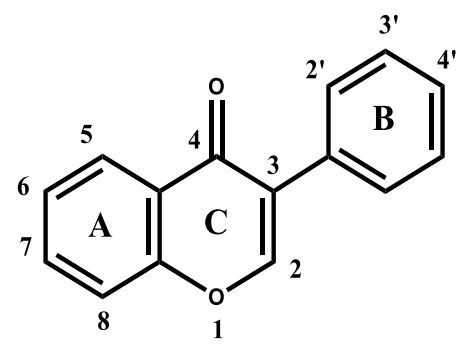

Fig. 1. 2D chemical structure of isoflavone nucleus

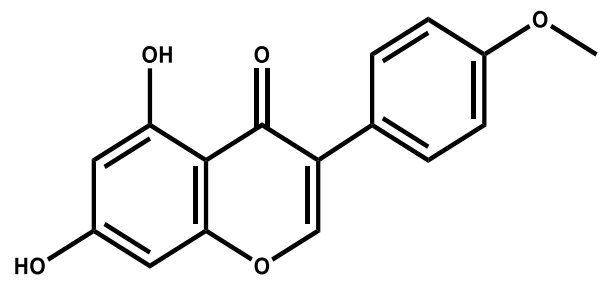

Fig. 2. 2D chemical structure of Biochanin-A

Several preclinical in vitro as well as in vivo studies on the anticancer properties of BiochaninA showed that this agent acts through a diversity of mechanisms such as cell cycle arrest and apoptosis in addition to possessing antiangiogenic effects [6]. Although Biochanin-A appears as a promising nature-derived anticancer agent, there is a paucity of information regarding specific target validation studies. In the current study, we used two online servers for computational prediction of physicochemical properties, ADME parameters, pharmacokinetic properties, and drug-likeness of Biochanin-A. These tools include both SwissADME and pkCSM servers. Furthermore, Cyclin-dependent kinase (CDK) 4/6 inhibitors have evolved as a revolutionary class of small molecule inhibitors for the management of breast cancer [8]. Those agents include abemaciclib, palbociclib as well as ribociclib [8]. Such agents inhibit G1/S cell cycle transition and afford better control over cancer progression [8]. Tyrosine kinase inhibitors of vascular endothelial growth factor receptor 2 (VEGFR-2) represent a key strategy for the management of invasive cancers by cutting down the tumor blood supply and inducing central necrosis [9]. Sorafenib is one of the famous multi-tyrosine kinase inhibitors that target VEGFR-2 as well as other tyrosine kinases and is highly efficient in the management of HCC [9, 10]. Treatment with agents from either class imposes several dose-limiting adverse reactions that undermine the quality of life of cancer patients [11]. Therefore, we performed in silico molecular docking studies for Biochanin-A as a potential dual hit for the VEGFR-2 and CyclinD1- CDK4 complex to validate the utility of Biochanin-A as a scaffold for the synthesis of efficient anticancer agents.

\section{MATERIALS AND METHODS}

\subsection{Biochanin-A properties prediction using online tools}

ADMET properties were generated using two servers, SwissADME (http://www.swissadme.ch) and

$\mathrm{PkCSM}$

(http://biosig.unimelb.edu.au/pkcsm/.) 


\subsection{In Silico Molecular Docking Studies}

Initially, the chemical structures of Biochanin$A$ and reference inhibitors were collected from PubChem [12] and converted into PDBQT format utilizing OpenBabel [13]. For VEGFR-2, the selected reference inhibitors were Axitinib, Cabozantinib, Pazopanib, and Sunitinib while Abemaciclib, Alvocidib, Palbociclib, and Riviciclib were selected for the CCND1-CDK4 complex. Crystal structures of the VEGFR-2 and CCND1-CDK4 complex were restored from the protein data bank (www.rcsb.org) [14]. For VEGFR-2, 4ASD crystal structure was selected over the higher resolution 3WZE due to higher temperature factor average while 2W96 crystal structure was used for the CCND1-CDK4 complex and the active site was located through alignment with 5L2T crystal structure of human CDK6-Ribociclib complex [15]. Selected proteins were prepared for molecular docking by removing solvent molecules and extracting the co-crystallized inhibitor compound for 4ASD using PyMOL [16], then saving the extracted ligands and clean protein molecules into separate PDB files. Finally, the clean protein molecules were protonated and converted to PDBQT format, and the grid dimensions files were also generated; all using AutoDock Tools [17].

Biochanin-A together with the additional reference inhibitors were docked to the previously prepared protein PDBQT files of both 4ASD and 2W96 using AutoDock Vina [18] after validating the docking protocol for both targets. This validation is based on redocking of the cocrystallized Sorafenib for 4ASD, and crossdocking of the co-crystallized Ribociclib of 5L2T to the $2 \mathrm{~W} 96$ protein complex. The visualization of the docked poses \& root-mean-square deviation (RMSD) calculations were performed using Discovery Studio Visualizer [19].

\subsection{In vitro studies}

\subsubsection{Chemicals}

Bio-A, sulforhodamine-B (SRB), and DMSO were purchased from Sigma-Aldrich (St. Louis, MO). Dulbecco's Modified Eagle Medium (DMEM), fetal bovine serum, and other cell culture materials were purchased from Lonza (Basel, Switzerland).

\subsubsection{Cell culture}

Human cancer cell lines of hepatocellular (HepG2), prostate (PC3), and breast (MCF-7) Cancers were obtained from Vacsera (Giza, Egypt). Cells were maintained in DMEM supplemented with $100 \mathrm{mg} / \mathrm{mL}$ of streptomycin, 100 units $/ \mathrm{mL}$ of penicillin, and $10 \%$ of heatinactivated fetal bovine serum in humidified, $5 \%$ (v/v) $\mathrm{CO}_{2}$ atmosphere at $37^{\circ} \mathrm{C}$.

\subsubsection{Cytotoxicity assay}

Cytotoxicity assays were performed according to the protocol [37]. Bio-A was dissolved in DMSO and kept at a stock concentration of 100 $\mathrm{mM}$. Cell viability was assessed by SRB assay. Aliquots of $100 \mu \mathrm{L}$ cell suspension $\left(5 \times 10^{3}\right.$ cells $)$ were in 96-well plates and incubated in complete media for $24 \mathrm{~h}$. Cells were treated with another aliquot of $100 \mu \mathrm{L}$ media containing drugs at various concentrations ranging from $(10 \mu \mathrm{m}, 100$ $\mu \mathrm{m})$. After $72 \mathrm{~h}$ of drug exposure, cells were fixed by replacing media with $150 \mu \mathrm{L}$ of $10 \%$ TCA and incubated at $4{ }^{\circ} \mathrm{C}$ for $1 \mathrm{~h}$. The TCA solution was removed, and the cells were washed 5 times with distilled water. Aliquots of $70 \mu \mathrm{L}$ SRB solution $(0.4 \% \mathrm{w} / \mathrm{v})$ were added and incubated in a dark place at room temperature for 10 min. Plates were washed 3 times with $1 \%$ acetic acid and allowed to air-dry overnight. Then, $150 \mu \mathrm{L}$ of TRIS $(10 \mathrm{mM})$ was added to dissolve protein-bound SRB stain; the absorbance was measured at $540 \mathrm{~nm}$ using a BMGLABTECH®-FLUO star Omega microplate reader (Ortenberg, Germany). 


\section{RESULTS}

\subsection{Biochanin-A properties prediction}

\subsubsection{BOILED-Egg diagram}

The results of the BOILED-Egg diagram suggest that the compound is orally available as is known about the compound. Also, it suggests that the compound will not cross the blood-brain barrier (BBB) and hence has limited central adverse effects (Fig. 3).

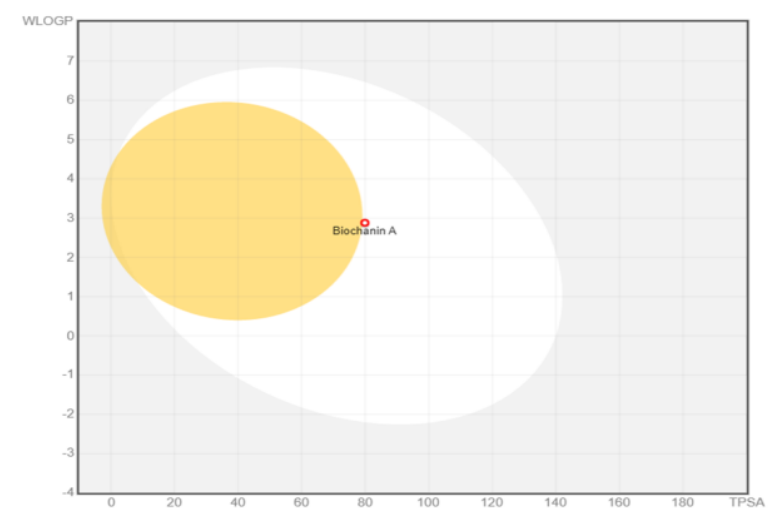

Fig.3. BOILED-Egg diagram generated by SwissADME server

\subsubsection{Swiss ADME report}

In the SwissADME report for Biochanin-A, a molar refractivity between 40-130 and TPSA lower than 90 means relatively low polarity and good oral bioavailability of Biochanin-A. Lipophilicity values are in the permissible range (-0.4-5.6) which implies a good lipophilicity profile for Biochanin-A. Besides, consensus log $\mathrm{P}_{\mathrm{O} / \mathrm{W}}$ (the average of all five lipophilicity predictions) is also in the permissible range. Furthermore, the water solubility report for Biochanin-A shows that it is mostly moderately soluble in water (near -4 value). The pharmacokinetic report for Biochanin-A showed that the gastrointestinal (GI) absorption is high with no $\mathrm{BBB}$ permeation similar to what was seen in the BOILED EGG diagram. Also, drug penetration to the skin is relatively low. Moreover, the physicochemical properties of
Biochanin-A obeyed the Lipinski rule of 5 without any violation, accompanied by a moderate bioavailability score that implies good oral bioavailability for Biochanin-A. Medicinal chemistry report for Biochanin-A showed zero alerts for PAINS and Brenk which suggests that Biochanin-A neither shows false positive biological screening results nor is putatively toxic. Furthermore, the rest of the report (lead likeness and synthetic accessibility) implies that Biochanin-A is suitable for optimization and can be easily synthesized (Table 1) [20].

\subsubsection{PkCSM report}

In the PkCSM report, the absorption parameters for Biochanin-A showed that it has relatively high Caco-2 permeability and can be absorbed in the intestine. However, it is poorly absorbed in the skin (low permeability). These results are similar to what was predicted by the SwissADME server. Biochanin-A was also found to be a substrate for P-glycoprotein but not an inhibitor for P-glycoprotein I or II. The distribution profile for Biochanin-A showed that it has a low volume of distribution, can moderately penetrate the CNS, and hardly crosses the blood-brain barrier (BBB) and finally, Biochanin-A can efficiently diffuse across cellular membranes.

Further, the metabolism report of BiochaninA revealed that it inhibits the majority of CYP 450 isoforms. Thus, it may result in drug-drug interactions when co-administered with other drugs by increasing their plasma concentration and in turn, their toxicity. The excretion profile for Bio-A implies its low total clearance and that it does not have the potential for adverse interactions with co-administered OCT2 inhibitors. Finally, the toxicity report for Biochanin-A showed that it is non-mutagenic, not an inhibitor for hERG I or II, doesn't cause any hepatotoxicity or skin sensation (Table 2) [21]. 
Table 1. Predicted Physicochemical and medicinal chemistry properties of Biochanin-A by Swiss-ADME server

\begin{tabular}{|c|c|}
\hline Property & Predicted value \\
\hline \multicolumn{2}{|l|}{ 1-Physicochemical properties } \\
\hline Formula & $\mathrm{C} 16 \mathrm{H} 12 \mathrm{O} 5$ \\
\hline Molecular weight & $284.26 \mathrm{~g} / \mathrm{mol}$ \\
\hline Number of heavy atoms & 21 \\
\hline Number of aromatic heavy atoms & 16 \\
\hline Fraction csp3 & 0.06 \\
\hline Number of rotatable bonds & 2 \\
\hline Number of Hydrogen bond acceptors & 5 \\
\hline Number Hydrogen bond donors & 2 \\
\hline Molar refractivity & 78.46 \\
\hline TPSA & $79.90 \mathrm{~A}^{2}$ \\
\hline \multicolumn{2}{|l|}{ 2- Lipophilicity } \\
\hline $\log \mathrm{P}_{\mathrm{O} / \mathrm{w}}$ (iLOGP) & 2.55 \\
\hline $\log \mathrm{P}_{\mathrm{O} / \mathrm{W}}(\mathrm{XLOGP} 3)$ & 2.99 \\
\hline $\log \mathrm{P}_{\mathrm{O} / \mathrm{W}}(\mathrm{WLOGP})$ & 2.88 \\
\hline Log $P_{\text {O/w }}$ (MLOGP) & 0.77 \\
\hline $\log \mathrm{P}_{\mathrm{O} / \mathrm{W}}($ SILICOS-IT $)$ & 3.03 \\
\hline Consensus $\log \mathrm{P}_{\mathrm{O} / \mathrm{w}}$ & 2.44 \\
\hline \multicolumn{2}{|l|}{ 3- water solubility } \\
\hline Log S (ESOL) & -3.92 \\
\hline Solubility & $3.43 \mathrm{e}-02 \mathrm{mg} / \mathrm{mL} ; 1.21 \mathrm{e}-04 \mathrm{~mol} / \mathrm{l}$ \\
\hline Class & Soluble \\
\hline Log S (Ali) & -4.33 \\
\hline Solubility & $1.32 \mathrm{e}-02 \mathrm{mg} / \mathrm{mL} ; 4.66 \mathrm{e}-05 \mathrm{~mol} / 1$ \\
\hline Class & Moderately soluble \\
\hline $\log$ S (SILICOS-IT) & -5.10 \\
\hline Solubility & 2.25e-03 milligram/milli; 7.91e-06 mol/1 \\
\hline Class & Moderately soluble \\
\hline \multicolumn{2}{|l|}{ 4- pharmacokinetics } \\
\hline Gastrointestinal absorption & High \\
\hline BBB permeant & No \\
\hline P-glycoprotein substrate & No \\
\hline Cytochrome P1A2 inhibitor & Yes \\
\hline Cytochrome $\mathrm{P} 2 \mathrm{C} 19$ inhibitor & No \\
\hline Cytochrome P2C9 inhibitor & No \\
\hline Cytochrome P2D6 inhibitor & Yes \\
\hline Cytochrome P3A4 inhibitor & Yes \\
\hline Logarithm $K_{P}$ (skin permeation) & $-5.91 \mathrm{~cm} / \mathrm{s}$ \\
\hline \multicolumn{2}{|l|}{ 5- Drug likeness } \\
\hline Lipinski & Yes: 0 violation \\
\hline Ghose & Yes \\
\hline Veber & Yes \\
\hline Egan & Yes \\
\hline Muegge & Yes \\
\hline Bioavailability Score & 0.55 \\
\hline \multicolumn{2}{|l|}{ 6- Medicinal Chemistry } \\
\hline PAINS & 0 alert \\
\hline Brenk & 0 alert \\
\hline Lead likeness & Yes \\
\hline Synthetic accessibility & 2.89 \\
\hline
\end{tabular}


Table 2. Predicted absorption, distribution, metabolism, excretion, and toxicity (ADMET) of Biochanin-A by PkCSM server

\section{1- Absorption}

Water solubility

Caco2 permeability

Intestinal absorption (human)

Skin permeability

P-glycoprotein substrate

P-glycoprotein I inhibitor

P-glycoprotein II inhibitor

\section{2- Distribution}

$\mathrm{VD}_{\mathrm{SS}}$ (human)

Fraction unbound (human)

BBB permeability

CNS permeability

\section{3- Metabolism}

Cytochrome P2D6 substrate

Cytochrome P3A4 substrate

Cytochrome P1A2 inhibitor

Cytochrome P2C19 inhibitor

Cytochrome P2C9 inhibitor

Cytochrome P2D6 inhibitor

Cytochrome P3A4 inhibitor

\section{4- Excretion}

Total Clearance

Renal OCT2 Substrate

\section{5- Toxicity}

AMES toxicity

Max. tolerated dose (human)

hERG I inhibitor

hERG II inhibitor

Oral Rat Acute Toxicity (LD50)

Oral Rat Chronic Toxicity (LOAEL)

Hepatotoxicity

Skin Sensation

T.Pyriformis toxicity

Minnow toxicity
$-3.735 \log \mathrm{mol} / \mathrm{L}$

0.897 log Papp in $10^{-6} \mathrm{~cm} / \mathrm{s}$

$93.028 \%$ absorbed

$-2.737 \log \mathrm{Kp}$

Yes

No

No

$-0.341 \log \mathrm{L} / \mathrm{kg}$

0.03

$-0.221 \log \mathrm{BB}$

$-2.115 \log$ PS

No

Yes

Yes

Yes

Yes

No

No

$0.247 \log \mathrm{ml} / \mathrm{min} / \mathrm{kg}$

No

No

$0.4 \log \mathrm{mg} / \mathrm{kg} /$ day

No

No

$1.851 \mathrm{~mol} / \mathrm{kg}$

$1.142 \log \mathrm{mg} / \mathrm{kg} \_$bw/day

No

No

$0.515 \log \mu \mathrm{g} / \mathrm{L}$

$0.681 \log \mathrm{mM}$

\subsection{In silico Molecular Docking Studies}

\subsubsection{Docking results of Cyclin-D1-CDK4}

The ATP binding domains of both CDK4 and CDK6 chains were found to align almost perfectly with only a slight variation in the depth of both pockets. CDK6 demonstrated a deeper pocket (Fig. 4). Upon docking was done with a large grid box that covers all the protein, ribociclib was found to favor binding to the ATP binding domain of the CDK4 subunit of the CCND1-CDK4 complex over all other pockets of 
this protein heterodimer. Ribociclib was also locally docked to both the CDK6 and CCND1CDK4 complex active sites, and the docked poses were compared with that of the cocrystallized pose of Ribociclib in the 5L2T model after its alignment to the 2W96 crystal structure using PyMOL (Fig. 5). The docked pose to the
5L2T model displayed only a minor spatial shift resulting in an RMSD of $0.22 \AA$. On the contrary, the docked pose to the 2W96 model demonstrated a relatively more significant spatial shift with an RMSD of $6.05 \AA$; because the ATP binding pocket of CDK4 is shallower.

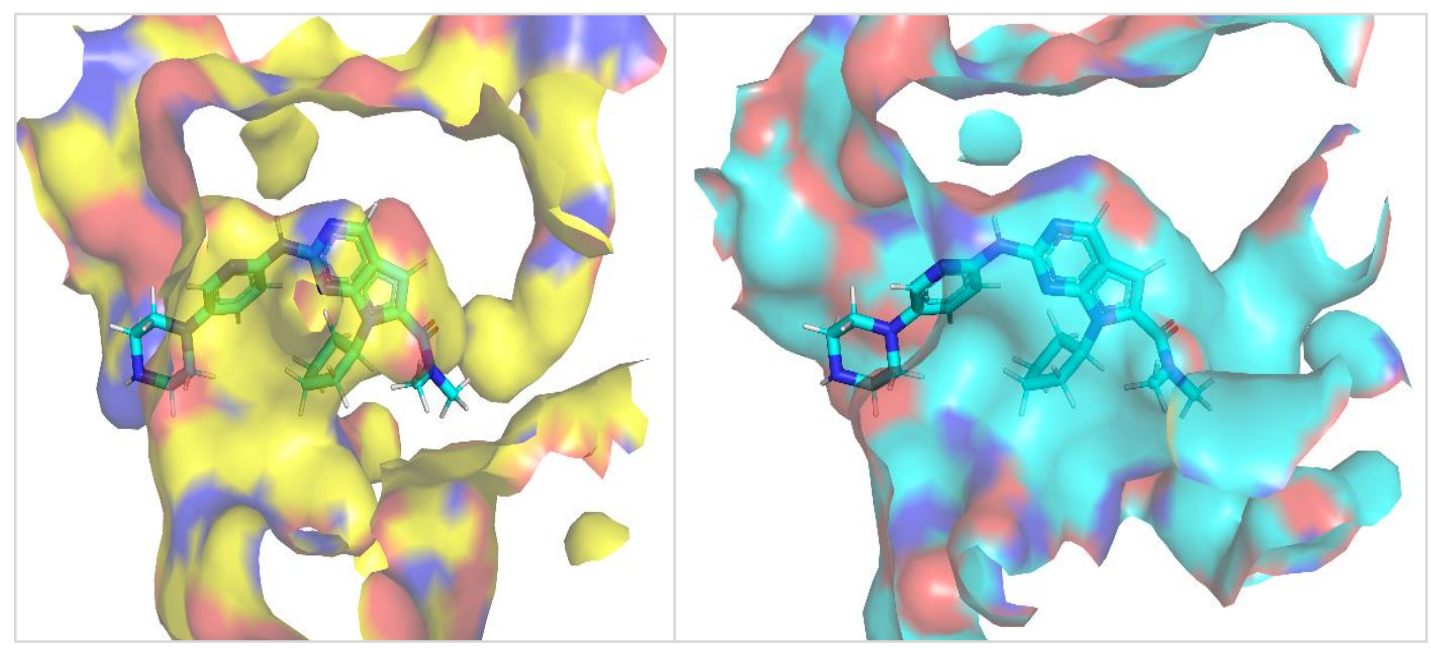

Fig.4. Binding site comparison using molecular surface representation of PyMOL depicting the ATP binding pockets of CDK4 on the left and CDK6 on the right. Ribociclib inhibitor molecule is displayed as sticks to demonstrate the relative depth of both pockets based on the alignment of 5L2T crystal structure to that of $2 \mathrm{~W} 96$

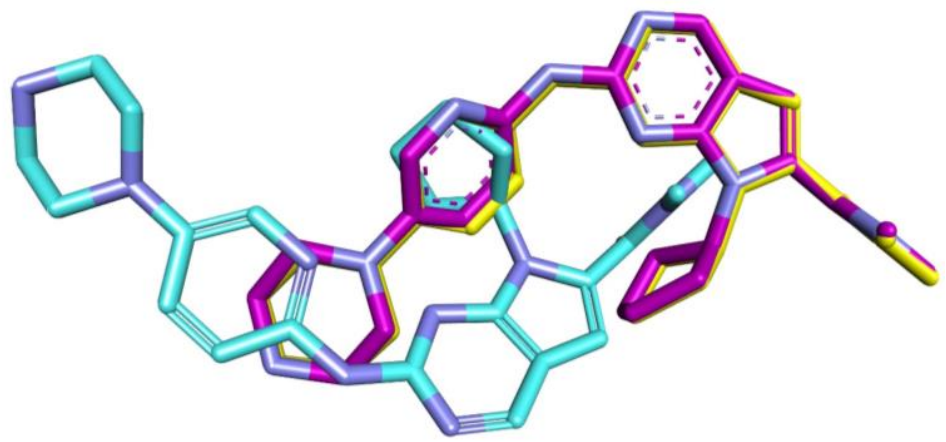

Fig.5. Generated poses from local docking of Ribociclib to the ATP binding domains of CDK6 (PDB ID: 5L2T) and CCND1 CDK4 complex (PDB ID: compared to the co crystallized Ribociclib residue of 5L2T after its alignment to the 2 W96 model; displayed respectively as canary yellow, cyan, and magenta sticks using Discovery Studio Visualizer

Biochanin-A was docked to the ATP binding site of the CDK4 subunit of the 2W96 model using the previously validated local docking protocol of Ribociclib. The docked pose with the highest score $(-8.3 \mathrm{kcal} / \mathrm{mol})$ was extracted from the PDBQT output file and visualized with the 2W96 protein complex using Discovery Studio Visualizer (Fig. 6). The carbon atom next to the cyclic oxygen of the isoflavone group within the docked Biochanin-A molecule displays a weak hydrogen bond interaction with the aspartate residue (ASP158) of the conserved DFG motif of the ATP binding domain; suggesting mild inhibition of the enzyme activity by blocking the metal ions' binding required for catalysis of phosphorylation process [15]. 
The docked pose of Biochanin-A was additionally validated through a comparison of the interactions with pocket amino acids of the ATP binding domain that belongs to the 5 reference inhibitor entities: Abemaciclib, Alvocidib, Palbociclib, Riviciclib, and Ribociclib. Upon the comparison, a significant consensus was noted concerning the interactions with Val20, Leu147, and Ile12. Moreover, Biochanin-A exhibits a hydrogen bond interaction with Lys 142 which also occurs in the binding mode of Abemaciclib. Scores of the best poses of Biochanin-A and reference inhibitors are shown in (Table 3).

Table 3. Predicted binding energy scores for the best poses generated from docking of reference inhibitors to the ATP binding pocket of CDK4 of the CCND1-CDK4 complex (PDB ID: 2W96)

\section{Compound Name}

\section{Predicted Binding Affinity (kcal/mol)}

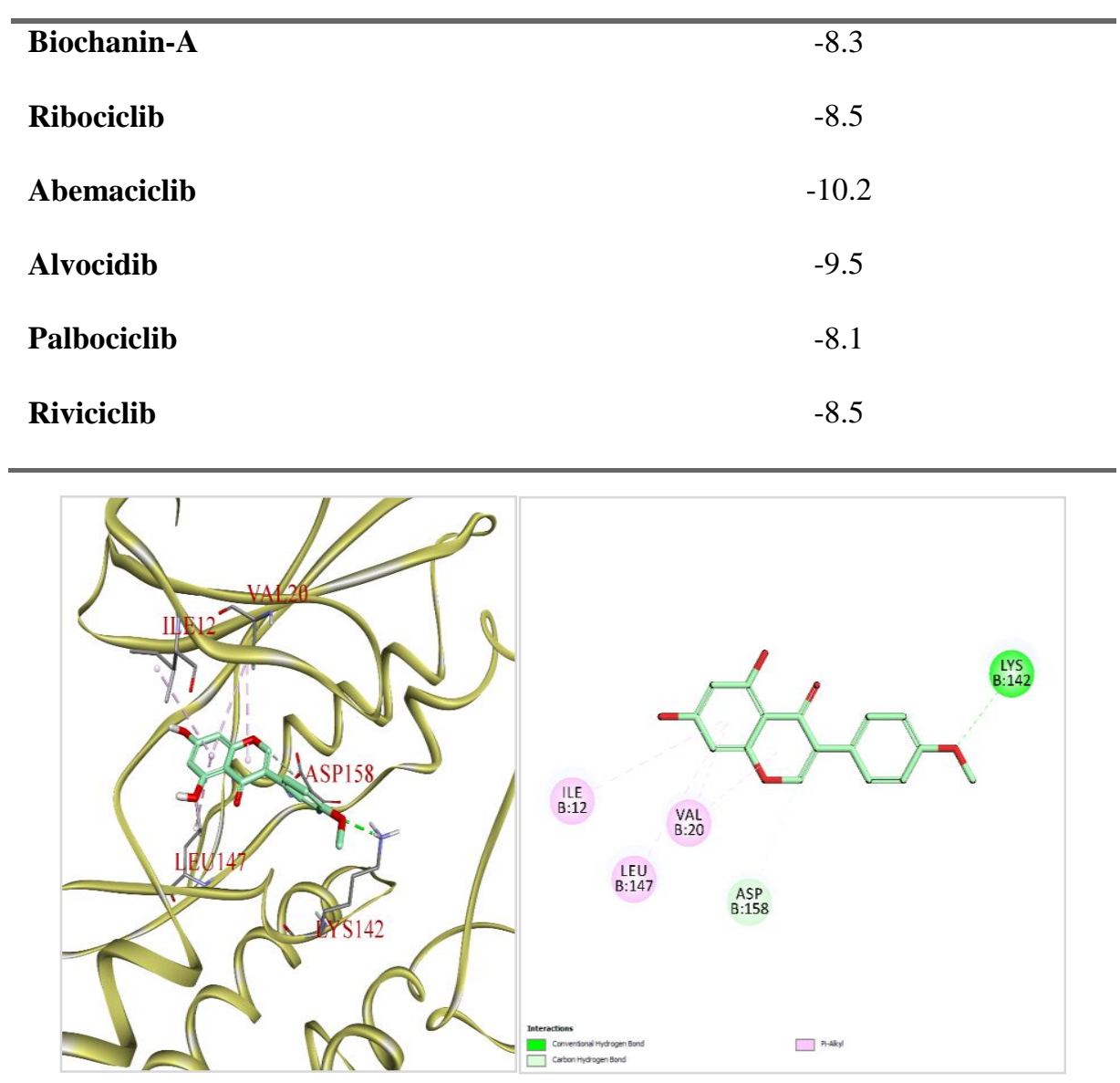

Fig.6. Generated pose from local docking of Biochanin A to the ATP binding domain of the CDK4 subunit of CCND1-CDK4 complex (PDB ID: 2W96). On the left, the binding mode is rendered in 3D with the ligand colored in faint green and the binding site residues labeled in red showing the residue name and residue identifier. On the right, the 2D interactions diagram is depicted with the legend shown at the bottom

\subsubsection{Docking results of VEGFR-2}

Sorafenib was redocked to the ATP binding domain of VEGFR-2 (4ASD PDB code). The sorafenib docked pose was found to have an RMSD value of $0.53 \AA$ compared to sorafenib crystal structure coordinates which validate the used docking protocol (Fig. 7). 


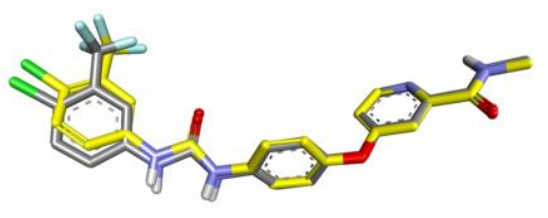

Fig.7. Generated pose from local re docking of Sorafenib to the ATP binding domain of VEGFR-2 (PDB ID: 4ASD) compared to the co crystallized binding mode of the Sorafenib residue within the 4ASD crystal structure; displayed respectively as canary yellow and silver sticks using Discovery Studio Visualizer
Biochanin-A (Fig. 8), as well as Axitinib, Cabozantinib, Pazopanib, and Sunitinib, were also docked to the ATP binding pocket belonging to the 4ASD model of VEGFR-2 using the same procedure. The predicted binding poses of tested compounds can be seen in. Moreover, the calculated binding energy score for the highestranking pose for tested compounds as well as Sorafenib is presented in (Table 4).

Table 4. Predicted binding energy scores for the best poses generated from docking of Biochanin-A and reference inhibitors to the ATP binding site of VEGFR-2 (PDB ID: 4ASD)

\section{Compound Name \\ Predicted Binding Affinity (kcal/mol)}

\begin{tabular}{lc}
\hline Biochanin-A & -8.6 \\
Sorafenib & -12.2 \\
Axitinib & -9.1 \\
Cabozantinib & -9.3 \\
Pazopanib & -7.2 \\
Sunitinib & -9.3 \\
\hline
\end{tabular}

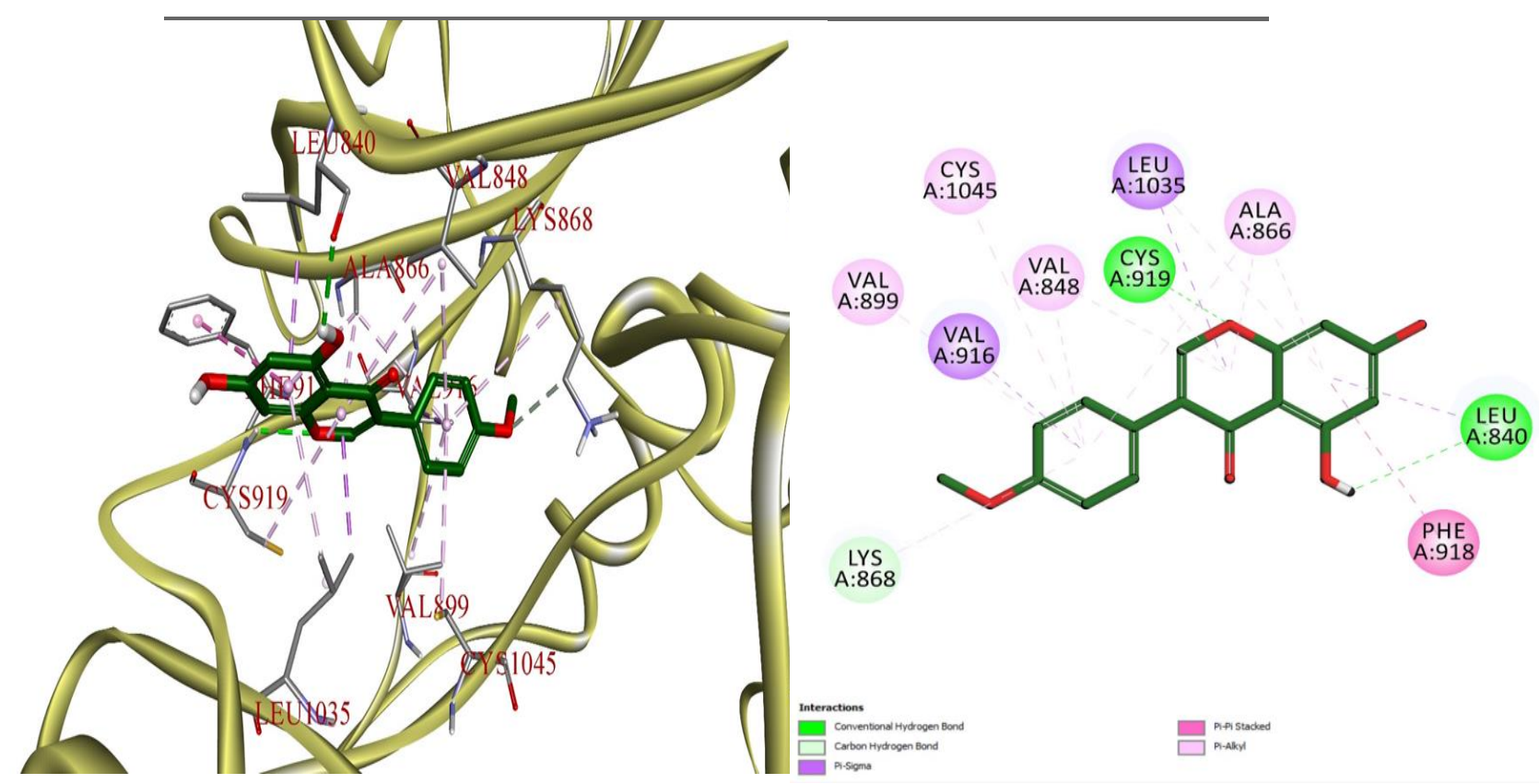

Fig.8. Generated pose from local docking of Biochanin A to the ATP binding pocket of VEGFR-2 (PDB ID: 4ASD). On the left, the binding mode is rendered in $3 \mathrm{D}$ with the ligand colored in green and the binding site residues labeled in red showing the residue name and residue identifier. On the right, the $2 \mathrm{D}$ interactions diagram is depicted with the legend shown at the bottom 


\subsection{In Vitro cytotoxicity of Bio-A on different cancer cells}

To investigate the cytotoxic effect of Bio-A in different cancer cells, concentration-response curves of Bio-A in HepG2, MCF-7, and PC-3 cell lines were assessed. In the HepG2 cell line, Bio-A at a concentration of $10 \mu \mathrm{M}$ induced a decrease in cell viability by $30.37 \%$, while at 100 $\mu \mathrm{M}$, Bio-A decreased viability by $92 \%$. In the MCF-7 cell line, Bio-A at a concentration of 10 $\mu \mathrm{M}$ induced a decrease in cell viability by $28.04 \%$, while at $100 \mu \mathrm{M}$, Bio-A decreased viability by $87.3 \%$. Furthermore, in a PC-3 cell line, Bio-A at a concentration of $10 \mu \mathrm{M}$ induced a decrease in cell viability by $0.34 \%$, while at 100 $\mu \mathrm{M}$, Bio-A decreased viability by $87.46 \%$ (Fig. 9).

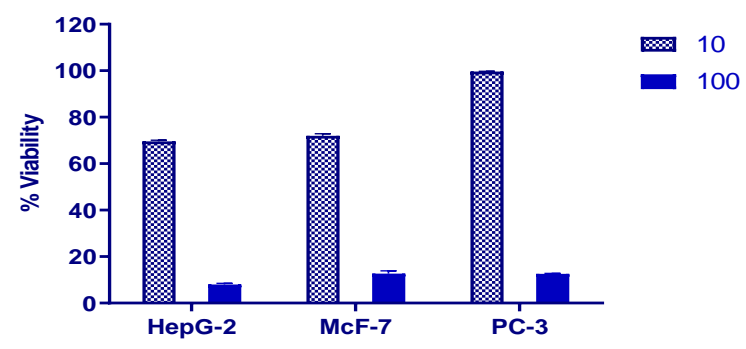

Fig.9. concentration-response plot of Biochanin-A in HepG2, McF-7 and PC-3 cell lines after $72 \mathrm{~h}$ of treatment. Data are expressed as means $\pm \mathrm{SD}(\mathrm{n}=6)$

\section{DISCUSSION}

Biochanin-A is marketed for the alleviation of menopausal symptoms [7]. Biochanin-A is a natural isoflavone that is found in red clover, soybean, peanut, and chickpeas [5]. This natural agent is available in a tablet dosage form as a food supplement (promensil $^{\circledR}$ ) and is used clinically for the management of postmenopausal symptoms due to its phytoestrogenic nature [6]. For a drug to exert a significant effect, its molecule should first reach the sickened organ in the body with a suitable dose concentration. Second, the drug with sufficient dose should remain in its bioactive form for a time long enough to induce its biological effect. The process of drug development is an early step in drug discovery. This step includes the estimation of the drug absorption, distribution, metabolism, and excretion or what is called ADME properties. At the drug development stage, the compounds under study are plentiful while access to physical samples of those drugs is infinitesimal [22]. Computer models are now considered an approach to mitigate the aforementioned issue. As computer models can substitute experimental procedures. Moreover, in the drug discovery phase, it was reported that the possibility of pharmacokinetics-related failure in the clinical phases can be reduced drastically by early assessment of ADME properties of the drug [23]. Thus, SwissADME is used as a web tool that offers free access to a gathering of rapid yet

BOILED-Egg model was generated Using SwissADME server, and values of the topological polar surface area (TPSA) and lipophilicity (WLOGP) properties of Biochanin$\mathrm{A}$ are $79.90 \mathrm{~A}^{2}$ and 2.88 , respectively which suggest that the compound will be orally absorbed without crossing BBB (Fig. 3). In addition, the SwissADME report showed the values related to different properties of Biochanin-A such as lipophilicity; the Physicochemical parameter that influences the affinity of a molecule towards binding sites, and passive transport through biological membranes [24].

Partition coefficient between water and an immiscible solvent is usually used for estimation of the molecules' affinity for a medium, and this affinity is calculated as a decimal logarithm of the partition coefficient $(\log \mathrm{P})$ in two nonmiscible solvents [25]. Water solubility; the measure of the amount of a substance (the solute) that can dissolve in water, pharmacokinetics; the study of the movement of xenobiotics (drugs/compounds/ new chemical entities (NCEs)) within the body after their 
administration [26]. Drug likeness is a parameter that is incorporated into the early stages of drug discovery. Besides, it is a qualitative property of chemicals determined by experts committee vote [27]. Furthermore, medicinal chemistry is a crucial parameter that is required to be determined during the early stage of drug discovery and development. The significance of medicinal chemistry is due to its ability to interpret the mechanism of action of biologically active compounds on a molecular basis. Moreover, medicinal chemists' interests extend to include not only bioactive compounds but also the identification and synthesis of their metabolic products [28]. The next step was to predict the ADMET properties of Biochanin-A using another server PkCSM (using graph-based signatures) to further investigate the possibility of Biochanin-A being an effective drug. PkCSM report revealed the predicted values for different parameters related to the ADMET properties of BiochaninA.

Regarding absorption of Biochanin A, water solubility was $(-3.735 \log \mathrm{mol} / \mathrm{L})$ indicating its moderate water solubility. Caco2 permeability (0.897) expresses the moderately high $\mathrm{Caco} 2$ permeability of Biochanin-A. Biochanin-A is well absorbed in the human intestine with an absorbance of $93.028 \%$. Skin permeability is a parameter of interest for the development of transdermal drug delivery. Biochanin-A has a moderate skin permeability with a $\log \mathrm{Kp}$ of 2.737. PkCSM report showed that Biochanin-A is likely to be a substrate for p-glycoprotein, without any likeness to be an inhibitor for Pglycoprotein I or II.

The volume of distribution (VD) is a pharmacokinetic property that is used to express a drug's tendency either to stay in the plasma or to be redistributed to other tissues. Mathematically, VD is the ratio between the amounts of the drug in the body to the plasma concentration of the drug at a specific time [29]. Distribution properties of Biochanin A showed that its volume of distribution (VDC) in humans is low (log VDss $=-0.341$ ), while the fraction of Biochanin-A that would be unbound in plasma is (0.03). Furthermore, Biochanin-A has moderate permeability across blood-brain barrier (BBB) $(\log \mathrm{BB}=-0.221)$ and across $\mathrm{CNS}(\log \mathrm{PS}=-$ 2.115).

Cytochrome P450 (CYP) is a hemeprotein that is mandatory for the metabolism of both drugs as well as xenobiotics [30]. Understanding the CYP system is crucial for advanced practitioners (APs) to avoid the profound consequences of drug-drug interactions [31]. Regarding Biochanin-A metabolism, Biochanin$\mathrm{A}$ is likely to be metabolized by CYP3A4 substrate but not CYP2D6 substrate. Furthermore, Biochanin-A is likely to be an inhibitor to different cytochrome $\mathrm{P} 450$ isoforms (CYP1A2, CYP2C19, CYP2C9), without any inhibitory action toward CYP2D6 and CYP3A4.

The organic cation transporter 2 (OCT2) is the transporter that intermediates the initial (basolateral) step in renal secretion of organic cations (positively charged molecules at physiological $\mathrm{pH}$ ) through human renal proximal tubules (RPTs). OCT2 exerts its action side by side with the apically expressed multidrug and toxin extruder transporters (MATEs) to play a central role in clearing the plasma of many cationic drugs as it boosts glomerular filtration [32]. As a result of OCT2 action, renal secretion plays a major role in the pharmacokinetics of numerous drugs [33]. The broad selectivity of OCTs, including OCT2, represents a major drawback as it makes them targets for unwanted drug-drug interactions (DDIs) [34]. Thus, the present focus of studies is mainly on the development of models with the advantage of predicting such interactions $[35,36]$. The PkCSM report of the present study showed that 
Biochanin-A is unlikely to be an OCT2 substrate with a total clearance of $(0.247 \mathrm{log} \mathrm{mL} / \mathrm{min} / \mathrm{kg})$.

The toxicity profile generated by the PkCSM server for Biochanin-A revealed that BiochaninA has negative AMES toxicity which suggests that Biochanin-A is not mutagenic or carcinogenic. The toxic dose threshold of Biochanin-A in humans is low as its highest tolerated dose is $(0.4 \mathrm{log} \mathrm{mg} / \mathrm{kg} /$ day $)$. Further, PkCSM reports showed that Biochanin-A doesn't have an inhibitory action on hERG I and II. Therefore, Biochanin-A as a compound doesn't lead to the development of long QT syndrome which results in a ventricular arrhythmia that could be fatal. Furthermore, the predicted value of the amount of Biochanin-A that causes the death of $50 \%$ of the population (LD50) is (1.851 $\mathrm{mol} / \mathrm{kg}$ ), while the lowest dose of Biochanin-A which leads to an adverse effect (LOAEL) is (1.142 log mg/kg BW/day). Moreover, the report revealed that Biochanin-A doesn't have either hepatotoxicity or skin sensation activities, which means that Biochanin-A is unlikely to be associated either with disruption to normal functions of the liver or with skin sensation and allergic contact dermatitis. Finally, the PkCSM report showed that Biochanin-A is toxic to $\mathrm{T}$. Pyryformis protozoa bacteria $(0.515 \log \mu \mathrm{g} / \mathrm{L})$. However, Biochanin-A did not have high acute toxicity against flathead minnows (0.681 log $\mathrm{mM}$ ) according to the PkCSM report.

The aberrant expression or activity of Cyclindependent kinases (CDK) is one of the hallmarks of several malignancies since CDKs are the key drivers of cell cycle progression and cell proliferation [37]. In 2017, the small molecule CDK4/6 inhibitors combined with endocrine therapy were approved as the standard of care for metastatic estrogen receptor (ER) positive breast cancer patients [38]. Preclinical studies showed the capacity of Biochanin-A to induce cell cycle arrest [6]. However, no data was reported regarding its effect on either Cyclin D1/CDK4 or CDK6 complexes. In the current work, the docking of Biochanin-A into the ATP binding domain of the Cyclin D1-CDK4 complex was performed through different steps. Firstly, Data retrieval and preparation for Biochanin-A and reference inhibitors of both Cyclin D1-CDK4 complex and VEGFR-2 to convert these chemical structures in PDBQT format using OpenBabel software. Secondly, performing binding site analysis and prediction using PyMol followed by global docking using AutoDock Vina to predict the most favorable binding pocket for Cyclin D1CDK4 complex. Finally, molecular docking simulation using Discovery Studio Visualizer for visualization of the docked poses and root-meansquare deviation (RMSD) calculations were performed. For the CyclinD1-CDK4 complex, binding site analysis revealed that CDK6 and CDK4 chains have perfect alignment to ribociclib (Fig. 4). Upon binding site analysis, the ATP binding domain of the CDK4 subunit of the CyclinD1-CDK4 complex was found to be the favorite binding site for ribociclib (Supporting Fig. S1). Afterward, the local docking of ribociclib to both CDK6 and CyclinD1-CDK4 complex resulted in an RMSD of $0.22 \AA$ for CDK6 and $6.05 \AA$ for CyclinD1-CDK4 complex because the ATP binding pocket of CDK4 is shallower (Fig. 5). The last step in the docking process was molecular docking of Biochanin-A to the ATP binding site of the CDK4 subunit of the 2W96 model using the previously validated local docking protocol of Ribociclib, followed by additional validation of the docked pose of Biochanin-A through comparison of the interactions with the pocket amino acids of the ATP binding domain to the reference inhibitors of cyclin-dependent kinase (CDK4/6) (Fig. 6). Docking of Biochanin-A to CyclinD1-CDK4 complex showed a hydrogen bond with Lys142 and Asp158 and several hydrophobic interactions with Ile12, Val20, and Leu147. It worth 
mentioning that Ribociclib also is forming a hydrogen bond with Asp163 in 5L2T PDB which aligns with Asp158 in CDK4 PDB 2W96. In addition, Biochanin-A showed a binding affinity of $(-8.3 \mathrm{kcal} / \mathrm{mol})$, which is comparable to its reference inhibitors as Ribociclib $(-8.5 \mathrm{kcal} / \mathrm{mol})$ and Palbociclib $(-8.1 \mathrm{kcal} / \mathrm{mol})$. Both agents are among the FDA-approved drugs for the management of advanced ER-positive breast tumors [39, 40] (Table 3) (Supporting Fig. S2S6).

The vascular endothelial growth factor (VEGF) is considered a major regulator of vascular development and angiogenesis within the tumor microenvironment [41]. VEGF acts through two key receptor tyrosine kinases, vascular endothelial growth factor receptor 1 (VEGFR-1) and VEGFR-2. VEGFR-2 is the main mediator of the VEGF signaling pathway in endothelial cell angiogenesis, invasion, and migration [42]. The antiangiogenic multi-tyrosine kinase inhibitors represent a cornerstone in the protocols for the management of invasive cancers [43]. Those agents include sorafenib, sunitinib, and axitinib which have been developed for the management of several advanced solid malignancies such as hepatocellular carcinoma and breast cancer [9]. In the current work, the insilico docking study was conducted to predict the binding affinity between Biochanin-A and VEGFR-2. Sorafenib was locally redocked to the ATP binding domain of the 4ASD model of the VEGFR-2. Afterward, Discovery Studio Visualizer showed acceptability of the docked pose of sorafenib for the local docking by having a relatively small RMSD value of $0.53 \AA$ (Fig. 7). Subsequently, Biochanin-A and five reference inhibitors were locally docked to the ATP binding pocket of the 4ASD model of the VEGFR-2. Afterward, by utilizing Discovery Studio Visualizer, the predicted poses with the strongest estimated binding affinities were extracted from their respective PDBQT output files and visualized with the protein part of the 4ASD crystal structure (Fig. 8). Finally, a molecular docking study revealed that BiochaninA docked into the VEGFR-2 receptor active site with a binding affinity $(-8.6 \mathrm{kcal} / \mathrm{mol})$ which is comparable to its standard inhibitors such as axitinib (-9.1 $\mathrm{kcal} / \mathrm{mol})$ and sunitinib (-9.3 $\mathrm{kcal} / \mathrm{mol})$. It is noteworthy that Biochanin-A binding affinity was at lower energy and more stable compared to that of the standard inhibitor pazopanib (-7.2 kcal/mol). However, the standard inhibitor sorafenib showed the best binding affinity (-12.2 kcal/mol) (Supporting Fig. S7S11) (Table 4). This tyrosine kinase inhibitor is the first-line therapy for advanced/metastatic hepatocellular carcinoma $[9, \mathbf{1 0}, 44]$. It is important to mention here that although Biochanin-A has shown got docking scores, it did not show interactions with Asp1046 and Glu885 which are known to be important interactions for VEGFR-2 inhibitors

Upon visual analysis of the binding mode of Biochanin-A, the cyclic oxygen of the isoflavone scaffold of Biochanin-A displayed a strong hydrogen bond interaction with the proton of the peptide backbone of Cys919 of the hinge region of the kinase. Furthermore, the side chain of the conserved anchoring Lys868 of the upper lobe exhibited two interactions with the ligand; a hydrophobic Pi-Sigma interaction with the substituted phenyl moiety of the isoflavone nucleus, and a non-classical hydrogen bond interaction with the ethereal oxygen atom of the terminal methoxy group. Finally, the ligand demonstrated a series of diverse hydrophobic interactions with several residues of the ATP binding site of the enzyme.

In vitro studies were performed to investigate the biological effect of Biochanin-A as an anticancer agent. SRB cytotoxicity assays were conducted to express the cytotoxic effects of Biochanin-A on different cancer cells including 
hepatocellular carcinoma (Hepa-2), breast cancer (McF-7), and prostate cancer (PC-3) cell lines, in the aforementioned assays Biochanin-A was tested at two different concentrations, 10 and 100 $\mu \mathrm{M}$. the results showed that at $10 \mu \mathrm{M}$ concentration, Biochanin-A decreased cell viability in HepG-2 by $30.37 \%$, in $\mathrm{McF}-7$ by $28.04 \%$, and in PC-3 by $0.34 \%$. In sharp contrast, the decrease in cell viability following treatment with Biochanin-A at $100 \mu \mathrm{M}$ concentration was $92 \%$ in HepG-2, $87.3 \%$ in McF-7, and $87.46 \%$ in PC-3 (Fig. 9). The previous results demonstrate the anti-cancer activity of Biochanin-A on different cancer types.

\section{Conclusion}

In conclusion, first, the reports of SwissADME and PKCSM servers for BiochaninA expressed the physicochemical properties of Biochanin-A including lipophilicity, absorption, distribution, metabolism, and excretion. These results suggest the susceptibility of Biochanin-A to be an effective drug. Second, the in-silico data suggests that Biochanin-A interacts with both VEGFR-2 and Cyclin D1-CDK4 complex with a docking affinity that is comparable to their standard inhibitors. These promising preliminary findings require further verification through kinase assays. Moreover, it encourages the use of Biochanin-A as a scaffold to generate more optimized nature-inspired inhibitors that have dual inhibitory functions against those key targets and carry a higher safety profile.

\section{Recommendation}

Further research is recommended to investigate other possible mechanisms of biochanin-A as an anticancer drug. This research includes, first, in-vivo studies for Biochanin-A for investigating the anticancer activity of Biochanin-A on mice. Second, in-vitro studies to investigate the possible mechanisms beyond the anticancer activity of Biochanin-A on different cell lines. Third, further in-silico studies for Biochanin-A on different tumor targets other than Cyclin-D1 or VEGFR-2 to explore any other mechanisms for Biochanin-A to exert its anticancer activity.

\section{Declarations}

\section{Ethics approval and consent to participate}

Not applicable

\section{Consent to publish}

Not applicable

\section{Availability of data and materials}

All data generated or analyzed during this study are included in this published article in the main manuscript.

\section{Competing interests}

No competing interests were declared by the authors.

\section{Funding statement}

No funding source was received

\section{Abbreviations}

VEGFR-2, Vascular endothelial growth factor 2; CDK, Cyclin-dependent kinase; HCC, Hepatocellular carcinoma; ADME, Absorption, distribution, metabolism and excretion; PDB, Protein data bank; RMSD, Root-mean-square deviation; ATP, Adenosine triphosphate; DFG motif, Asp-Phe-Gly motif; NCE, New chemical entities; VD, Volume of distribution; BBB, Blood-brain barrier; CYP, Cytochrome P; Aps, Advanced practitioners; OCT2, Organic cation transporter 2; RPT, Renal proximal tubules; DDI, Drug-drug interactions; hERG, Human-ether-ago-go gene; FDA, Food and Drug Agency.

\section{REFERENCES}

1. Ko K. P. (2014). Isoflavones: chemistry, analysis, functions, and effects on health and cancer. Asian Pacific journal of cancer 
prevention: APJCP, 15(17), 7001-7010. DOI: 10.7314/apjcp.2014.15.17.7001

2. Cassady JM, Zennie TM, Chae YH, Ferin MA, Portuondo NE et al. (1988) Use of a mammalian cell culture benzo (a) pyrene metabolism assay for the detection of potential anticarcinogens from natural products: inhibition of metabolism by biochanin $\mathrm{A}$, an isoflavone from Trifolium pratense L. Cancer Res 48(22): 6257-6261. PMID: 3180045.

3. Oh JS, Cho IA, Kang KR, You JS, Yu SJ, et al. (2016) Biochanin-A antagonizes the interleukin-1 $\beta$-induced catabolic inflammation through the modulation of $\mathrm{NF \kappa B}$ cellular signaling in primary rat chondrocytes. Biochemical and Biophysical Research Communications 477: 723-730. DOI: $10.1016 /$ j.bbrc.2016.06.126.

4. Heinonen $S$, Wähälä $K$, Adlercreutz H JAB (1999) Identification of isoflavone metabolites dihydrodaidzein, dihydrogenistein, 6'-OH-Odma, and cis-4-OH-equol in human urine by gas chromatography-mass spectroscopy using authentic reference compounds. 274(2): 211219. DOI: 10.1006/abio.1999.4279.

5. Yu, C., Zhang, P., Lou, L., \& Wang, Y. (2019). Perspectives Regarding the Role of Biochanin A in Humans. Frontiers in pharmacology, 10, 793. https://doi.org/10.3389/fphar.2019.00793

6. Sarfraz, A., Javeed, M., Shah, M. A., Hussain, G., Shafiq, N., Sarfraz, I., Riaz, A., Sadiqa, A., Zara, R., Zafar, S., Kanwal, L., Sarker, S. D., \& Rasul, A. (2020). Biochanin A: A novel bioactive multifunctional compound from nature. The Science of the total environment, 722 ,

137907. https://doi.org/10.1016/j.scitotenv.2020.13797

7. Booth NL, Piersen CE, Banuvar S, Geller SE, Shulman LP, et al. (2006) Clinical studies of red clover (Trifolium pratense) dietary supplements in menopause: a literature review. Menopause 13(2): 251-264. DOI: 10.1097/01.gme.0000198297.40269.f7.

8. Niu Y, Xu J, Sun T (2019) Cyclin-dependent kinases 4/6 inhibitors in breast cancer: Current status, resistance, and combination strategies. 10(22): 5504-5517. DOI: 10.7150/jca.32628.

9. Niu G, Chen X J C (2010) Vascular endothelial growth factor as an antiangiogenic target for cancer therapy. Curr Drug Targets 11(8): 1000-1017.

DOI: $10.2174 / 138945010791591395$.

10. Tiffany Sandra Wai Ling Khoo, Arif Rehman, John K. Olynyk (2019) Tyrosine Kinase Inhibitors in the Treatment of Hepatocellular Carcinoma. Hepatocellular Carcinoma 127-139. DOI: 10.15586/hepatocellularcarcinoma.2019. $\operatorname{ch} 7$

11. Ho VWT, Tan HY, Wang N, Feng Y (2019) Cancer Management by Tyrosine Kinase Inhibitors: Efficacy, Limitation, and Future Strategies in Tyrosine Kinases as Druggable Targets in Cancer, Intech Open. DOI: 10.5772/intechopen.82513.

12. Kim S, Thiessen PA, Bolton EE, Chen J, Fu $G$, et al. (2016) PubChem substance and compound databases. 44(D1): D1202-D1213. DOI: $10.1093 / \mathrm{nar} / \mathrm{gkv} 951$.

13. O’Boyle NM, Banck M, James CA, Morley C, Vandermeersch T et al. (2011) Open Babel: An open chemical toolbox 33(3). DOI: 10.1186/1758-2946-3-33.

14. Berman HM, Westbrook J, Feng Z, Gilliland G, Bhat TN, et al. (2000) The protein data bank. Nucleic Acids Res 28(1): 235-242. DOI: 10.1093/nar/28.1.235.

15. Martin MP, Endicott JA, Noble ME (2017) Structure-based discovery of cyclin-dependent protein kinase inhibitors. Essays Biochem 61(5): 439-452. DOI: 10.1042/EBC20170040. 
16. Schrödinger L (2010) The PyMOL molecular graphics system, version $1.3 \mathrm{r} 1$. Scientific Research.

17. Sanner M, Huey R, Dallakyan S, Karnati S, Lindstrom W, et al. (2007) AutoDockTools, version 1.4. 5. The Scripps Research Institute, La Jolla.

18. Trott O, Olson A J (2010) AutoDock Vina: improving the speed and accuracy of docking with a new scoring function, efficient optimization, and multithreading. J Comput Chem 31(2): 455-461. DOI: $10.1002 /$ jcc. 21334

19. Biovia DS (2017) Discovery studio visualizer, San Diego, CA, USA. pp. 936.

20. 46. Daina, A., Michielin, O., \& Zoete, V. (2017). SwissADME: a free web tool to evaluate pharmacokinetics, drug-likeness, and medicinal chemistry friendliness of small molecules. Scientific reports, 7, 42717. https://doi.org/10.1038/srep42717

21. Pires, D. E., Blundell, T. L., \& Ascher, D. B. (2015). pkCSM: Predicting Small-Molecule Pharmacokinetic and Toxicity Properties Using Graph-Based Signatures. Journal of medicinal chemistry, 58(9), 4066-4072. https://doi.org/10.1021/acs.jmedchem.5b0010 4

22. Dahlin, J. L., Inglese, J. \& Walters, M. A. Mitigating risk in academic preclinical drug discovery. Nature Rev. Drug Discov. 14, 279294 (2015).

23. Hay, M., Thomas, D. W., Craighead, J. L., Economides, C. \& Rosenthal, J. Clinical development success rates for investigational drugs. Nature Biotechnol. 32, 40-51 (2014).

24. Jevri'c, L.R.; Karadži'c, M.Ž.; Mandi'c, A.I.; Podunavac Kuzmanovi'c, S.O.; Kova cevi'c, S.Z.; Nikoli'c, A.R.; Oklješa, A.M.; Saka č, M.N.; Penov Gaši, K.M.; Stojanovi'c, S.Z.
Lipophilicity estimation and characterization of selected steroid derivatives of biomedical importance applying RP HPLC. J. Pharm. Biomed. Anal. 2017, 134, 27-35. [CrossRef]

25. Klose, M.; Theiner, S.; Varbanov, H.; Hoefer, D.; Pichler, V.; Galanski, M.; Meier-Menches, S.; Keppler, B. Development and validation of liquid chromatography-based methods to assess the lipophilicity of cytotoxic Platinum (IV) complexes. Inorganics 2018, 6, 130. [CrossRef]

26. Fan, J., \& de Lannoy, I. A. (2014). Pharmacokinetics. Biochemical pharmacology, 87(1), 93-120. https://doi.org/10.1016/j.bcp.2013.09.007

27. O. Ursu, A. Rayan, A. Goldblum, T. Oprea, Understanding drug-likeness, Wiley Interdiscip. Rev. Comput. Mol. Sci 5 (2011) 760-781, https://doi.org/10.1002/wcms.52.

Doi: $10.1002 /$ wcms.52

28. Wermuth, C. G., Ganelin, C. R., Lindberg, P., Mitscher, L. A. Glossary of terms used in medicinal chemistry (IUPAC Recommendations, 1997). In Annual Reports in Medicinal Chemistry (Adam, J., Ed.). Academic Press: San Diego, CA, 1998, pp. $385-395$.

29. Oie S. Drug distribution and binding. J Clin Pharmacol. 1986 Nov-Dec; 26(8):583-6. [PubMed] [Reference list]

30. Estabrook Ronald W. A passion for P450s (remembrances of the early history of research on cytochrome P450). Drug metabolism and disposition: the biological fate of chemicals. 2003; 31:1461-1473. [PubMed] [Google Scholar]

31. McDonnell, A. M., \& Dang, C. H. (2013). Basic review of the cytochrome p450 system. Journal of the advanced practitioner in oncology, 4 263-268. 
https://doi.org/10.6004/jadpro.2013.4.4.7

DOI: 10.6004/jadpro.2013.4.4.7

32. Scotcher D, Jones C, Posada M, Gallatin A, and Rostami-Hodjegan A. Key to Opening Kidney for In Vitro-In Vivo Extrapolation Entrance in Health and Disease: Part II: Mechanistic Models and In Vitro-In Vivo Extrapolation. The AAPS Journal 18: 10821094, 2016.

33. Lepist EI, and Ray AS. Renal drug-drug interactions: what we have learned and where we are going. Expert Opin Drug Metab Toxicol 8: 433-448, 2012.

34. 124. Xu Y, Liu X, Li S, Zhou N, Gong L, Luo C, Luo X, Zheng M, Jiang H, and Chen $\mathrm{K}$. Combinatorial pharmacophore modeling of organic cation transporter 2 (OCT2) inhibitors: insights into multiple inhibitory mechanisms. Mol Pharm 10: 4611-4619, 2013.

35. Ivanyuk A, Livio F, Biollaz J, and Buclin T. Renal drug transporters and drug interactions. Clin Pharmacokinet 56: 825-892, 2017.

36. Sandoval PJ, Zorn KM, Clark AM, Ekins S, and Wright SH. Assessment of substratedependent ligand interactions at the Organic Cation Transporter OCT2 Using six model substrates. Mol Pharmacol 94: 1057-1068, 2018.

37. Ding L, Cao J, Lin W, Chen H, Xiong X, et al. (2020) The Roles of Cyclin-Dependent Kinases in Cell-Cycle Progression and Therapeutic Strategies in Human Breast Cancer. $21(6)$ : 1960. DOI: $10.3390 / \mathrm{ijms} 21061960$

38. Pernas S, Tolaney SM, Winer EP, Goel S (2018) CDK4/6 inhibition in breast cancer: current practice and future directions. 10:1758835918786451. DOI: $10.1177 / 1758835918786451$
39. Sobhani N, D'Angelo A, Pittacolo M, Roviello G, Miccoli A, et al. (2019) Updates on the CDK4/6 inhibitory strategy and combinations in breast cancer. Cells 8(4): 321. DOI: $10.3390 /$ cells 8040321

40. Bellet M, Ahmad F, Villanueva R, Valdivia C, Palomino-Doza J, et al. (2019) Palbociclib and ribociclib in breast cancer: consensus workshop on the management of concomitant medication. Therapeutic Advances in Medical Oncology 11. DOI: $10.1177 / 1758835919833867$.

41. Lee SH, Jeong D, Han YS, Baek MJ (2015) Pivotal role of vascular endothelial growth factor pathway in tumor angiogenesis. 89(1): 1-8. DOI: 10.4174/astr.2015.89.1.1

42. Napione L, Alvaro M, Bussolino F (2017) VEGF-mediated signal transduction in tumor angiogenesis. pp. 227. DOI: 10.5772/66764

43. Gotink KJ, Verheul H M J A (2010) Antiangiogenic tyrosine kinase inhibitors: what is their mechanism of action? 13(1): 1-14. DOI: 10.1007/s10456-009-9160-6.

44. Li D, Sedano S, Allen R, Gong J, Cho M (2019) Current treatment landscape for advanced hepatocellular carcinoma: Patient outcomes and the impact on quality of life. Cancers (Basel) 11(6): 841. DOI: $10.3390 /$ cancers 11060841 